arXiv: arXiv:0000.0000

\title{
Wald Statistics in high-dimensional PCA
}

\author{
Matthias Löffler \\ Statistical Laboratory, Centre for Mathematical Sciences, University of Cambridge, \\ Wilberforce Road, CB3 OWB Cambridge, United Kingdom. e-mail: \\ m.loffler@statslab.cam.ac.uk
}

\begin{abstract}
In this note we consider PCA for Gaussian observations $X_{1}, \ldots, X_{n}$ with covariance $\Sigma=\sum_{i} \lambda_{i} P_{i}$ in the 'effective rank' setting with model complexity governed by $\mathbf{r}(\Sigma):=\operatorname{tr}(\Sigma) /\|\Sigma\|$.

We prove a Berry-Essen type bound for a Wald Statistic of the spectral projector $\hat{P}_{r}$. This can be used to construct non-asymptotic confidence ellipsoids and tests for spectral projectors $P_{r}$. Using higher order pertubation theory we are able to show that our Theorem remains valid even when $\mathbf{r}(\Sigma) \gg \sqrt{n}$.
\end{abstract}

Keywords and phrases: PCA, Spectral projectors, Central Limit Theorem, confidence sets.

\section{Introduction}

Principal Component Analysis (PCA) is a widely used dimension-reduction technique in statistics. In the traditional Gaussian setting going back to Anderson [1] one observes $n$ i.i.d. zero mean Gaussian random variables with covariance matrix $\Sigma$. In more recent years mainly three working assumptions on $\Sigma$ have been considered: The spiked covariance model, the spiked sparse covariance model and the 'effective rank' setting.

Johnstone [6] introduced the spiked covariance model in which $\Sigma$ is given by

$$
\Sigma=\sum_{i=1}^{l} s_{i} \theta_{i} \theta_{i}^{T}+\sigma^{2} I_{p} .
$$

Subsequent work in this model has mainly been focused on the failures of PCA in high-dimensions when the dimension $p \rightarrow \infty$ and $p / n \rightarrow$ const. [7, 15, 13, 21]. A remedy is to assume that the leading eigenvectors $\theta_{i}$ in (1.1) are sparse, enabling thus inference even when $p / n \rightarrow \infty[3,5,18,2,20]$.

We will consider the effective rank setting [16, 12, 8, 10, 9, 14]. Here no assumptions on the particular structure of $\Sigma$ are made, except that the effective rank $\mathbf{r}(\Sigma):=\operatorname{tr}(\Sigma) /\|\Sigma\|=o(n)$ where $\operatorname{tr}(\cdot)$ denotes the trace and $\|\cdot\|$ the operator norm. This allows for a wider range of models, for example $\Sigma$ with a polynomial or exponential decay of the eigenvalues [16].

Rates of convergence and limiting results for empirical spectral projectors and empirical eigenvectors were proven in [8]. Building upon this [12] proved that a de-biased empirical eigenvector attains the semi-parametric efficiency bound. 
A method for constructing Frobenius type confidence sets for $P_{r}$ was given in the two papers [10] and [11]. However, their approach requires sample splitting into three samples and the assumption that $\|\Sigma\|_{F}^{2} \rightarrow \infty$. The latter is not necessarily fulfilled, for example in a decaying eigenvector model where the eigenvalues $\lambda_{i}$ scale as $i^{-\alpha}, \alpha>1 / 2$. Others approaches based on the bootstrap and Bayesian inference, respectively, were proposed by [14] and [17] but require at least $\mathbf{r}(\Sigma)=o\left(n^{1 / 3}\right)$ and do not deal with the harder case $\mathbf{r}(\Sigma) \gg \sqrt{n}$ where one needs to account for bias.

In this note we present a third possibility based on Wald Statistics for constructing a Frobenius type confidence set for $P_{r}$. We show that when $p \rightarrow \infty$ a further normalized Wald statistic of $\hat{P}_{r}-P_{r}$ is asymptotically Gaussian.

Our main contribution is that we show how to deal with the critical case $\mathbf{r}(\Sigma) \gg \sqrt{n}$ by using second order pertubation theory, requiring for example in the spiked covariance model (1.1) that $\mathbf{r}(\Sigma)=o\left(n^{2 / 3}\right)$.

\subsection{Set-up $\&$ notation}

For matrices $A, B, C$ we define the Kronecker product as $(A \otimes B) C=A C B^{T}$ and the Frobenius inner product $\langle A, B\rangle:=\operatorname{tr}\left(A^{T} B\right)$. $\|\cdot\|_{F}$ denotes the Frobeniusnorm. The notation $\|\cdot\|$ will be used for the operator norm and in slight abuse of notation for the Euclidean norm of vectors with corresponding Euclidean inner product $\|v\|:=\langle v, v\rangle:=v^{T} v$.

We will frequently use the following convention throughout the paper: for nonnegative $a, b a \lesssim b$ means that there exists a constant $C$ not depending on $n$ or $\mathbf{r}(\Sigma)$ such that $a \leq C b$.

We assume that $X_{1}, \ldots X_{n}$ are $i . i . d$ centred Gaussian vectors in $\mathbb{R}^{p}$ with $\mathbb{E}\|X\|^{2}<$ $\infty$. We denote by $\Sigma=\mathbb{E} X_{1} X_{1}^{T}$ the covariance matrix of the observations $X_{1}, \ldots, X_{n}$ and we denote the empirical covariance matrix by

$$
\hat{\Sigma}=: \frac{1}{n} \sum_{j=1}^{n} X_{j} X_{j}^{T} .
$$

We define the effective rank: $\mathbf{r}(\Sigma):=\operatorname{tr}(\Sigma) /\|\Sigma\|$.

Since $\Sigma$ is symmetric and positive semidefinite it has spectral decomposition $\Sigma=\sum_{s} \lambda_{s} P_{s}$ where $\lambda_{s}$ are distinct strictly positive, descending eigenvalues and $P_{s}$ are the corresponding spectral projectors. Let $\mu_{j}$ denote the eigenvalues of $\Sigma$ arranged in a non-increasing order and repeated with their multiplicities. Define $\Delta_{r}:=\left\{j: \mu_{j}=\lambda_{r}\right\}$. As described (and proven in Lemma 2.2.) in [12] it suffices to assume that $\Delta_{r}$ is known as $\Delta_{r}$ can be identified on an event of high probability under our assumptions.

We thus denote by $\hat{P}_{r}$ the spectral projector corresponding to the eigenvalues $\left\{\mu_{j}(\hat{\Sigma}), j \in \Delta_{r}\right\}$ and by $\hat{\lambda}_{r}$ one arbitrary chosen eigenvalue from the same set. We denote by $\bar{g}_{r}:=\min \left(\lambda_{r-1}-\lambda_{r}, \lambda_{r}-\lambda_{r+1}\right)$ the spectral gap of the eigenvalue $\lambda_{r}$ with the convention that $\lambda_{0}=\infty$. By $\lambda_{\min }$ we denote the smallest eigenvalue 
of $\Sigma$ and likewise by $\hat{\lambda}_{\text {min }}$ the smallest eigenvalue of $\hat{\Sigma}$. If $\operatorname{card}\left(\Delta_{r}\right):=m_{r}=1$ we define $\hat{\theta}_{r}$ to be a sample eigenvector belonging to the eigenvalue $\hat{\lambda}_{r}$.

\section{Main result}

Wald statistics [19] are commonly used when the dimension of the parameter space is $p=$ const. The Fisher information for the model $X \sim N(0, \Sigma)$ is $\mathbb{I}(\Sigma)=\frac{1}{2}\left(\Sigma^{-1} \otimes \Sigma^{-1}\right)$ (e.g. [4]). If $p$ is constant the maximum likelihood estimator $\hat{\Sigma}$ for $n$ i.i.d. observations is asymptotically Gaussian distributed with $\sqrt{n}$-rate and limiting covariance $\mathbb{I}(\Sigma)^{-1}=2(\Sigma \otimes \Sigma)$. Applying the delta method to $g(\Sigma):=P_{r}$ shows that $g(\hat{\Sigma})$ is asymptotically Gaussian, too, and has limiting covariance

$\mathbb{I}\left(P_{r}\right)^{-1}:=2 \sum_{s \neq r}\left(P_{s} \otimes P_{r}+P_{r} \otimes P_{s}\right) \frac{\lambda_{r} \lambda_{s}}{\left(\lambda_{r}-\lambda_{s}\right)^{2}}=2\left(\Sigma C_{r}^{2} \otimes P_{r} \Sigma+\Sigma P_{r} \otimes C_{r}^{2} \Sigma\right)$,

where we define the resolvent operator $C_{r}:=\sum_{s \neq r} \frac{P_{s}}{\lambda_{r}-\lambda_{s}}$.

If $p$ remains fixed and $\operatorname{rank}\left(P_{r}\right)=m_{r}$ it follows that the Wald statistic below converges to a $\chi^{2}$-distributed random variable,

$$
n\left\|\mathbb{I}\left(P_{r}\right)^{1 / 2}\left(\hat{P}_{r}-P_{r}\right)\right\|_{F}^{2} \stackrel{d}{\rightarrow} \chi_{m_{r}\left(p-m_{r}\right)}^{2},
$$

where we denote

$$
\mathbb{I}\left(P_{r}\right)^{1 / 2}=\frac{1}{\sqrt{2}}\left(\Sigma^{-1 / 2} C_{r}^{-1} \otimes P_{r} \Sigma^{-1 / 2}+\Sigma^{-1 / 2} P_{r} \otimes C_{r}^{-1} \Sigma^{-1 / 2}\right)
$$

and, slightly abusing notation, $C_{r}^{-1}:=\lambda_{r} I-\Sigma$.

In the high-dimensional regime with $p \rightarrow \infty$ the test statistic above is stochastically unbounded and thus the $\chi^{2}$-approximation becomes invalid. Hence one has to further normalize, eventually obtaining a Gaussian limit instead. Moreover, higher order terms do not simply vanish anymore as $n \rightarrow \infty$. Particularly, when applying $\mathbb{I}\left(P_{r}\right)^{1 / 2}$ to $\left(\hat{P}_{r}-P_{r}\right)$ one has to multiply with $\Sigma^{-1 / 2}$ and thus the size of higher order error terms depends on the smallest eigenvalue of $\Sigma$ which we denote by $\lambda_{\min }$.

Lemma 2.1. Suppose that $\boldsymbol{r}(\Sigma)=o(n)$ and that $\mathbb{E}\|\hat{\Sigma}-\Sigma\| \leq(1-\gamma) \bar{g}_{r} / 2$ for some $\gamma \in(0,1)$. For $g_{i}$ denoting i.i.d. standard Gaussian random variables we have that,

$$
\frac{n\left\|\mathbb{I}\left(P_{r}\right)^{1 / 2}\left(\hat{P}_{r}-P_{r}\right)\right\|_{F}^{2}-m_{r}\left(p-m_{r}\right)}{\sqrt{2 m_{r}\left(p-m_{r}\right)}} \stackrel{d}{=} \frac{\sum_{i=1}^{m_{r}\left(p-m_{r}\right)}\left(g_{i}^{2}-1\right)}{\sqrt{2 m_{r}\left(p-m_{r}\right)}}+Z_{n},
$$


where $Z_{n}$ fulfills with probability at least $1-e^{-t}$ for every $1 \leq t \leq n$

$$
\begin{aligned}
\left|Z_{n}\right| \leq & C\left(\gamma, \bar{g}_{r}, m_{r},\|\Sigma\|, \lambda_{r}\right) . \\
& \left(\frac{t}{\sqrt{n}} \bigvee \frac{(\boldsymbol{r}(\Sigma) \vee t) \sqrt{t}}{\lambda_{\min } \sqrt{n p}} \bigvee \sqrt{\frac{p}{n}} \sqrt{t} \bigvee \frac{t^{3 / 2}}{\sqrt{n p}} \bigvee \frac{\sqrt{p} \mathbf{r}(\Sigma)}{n} \bigvee \frac{\mathbf{r}(\Sigma)^{2}}{n \sqrt{p} \lambda_{\min }}\right) .
\end{aligned}
$$

The bounds on $\left|\mathbb{E}\left\|\mathbb{I}\left(P_{r}\right)^{1 / 2}\left(\hat{P}_{r}-P_{r}\right)\right\|_{F}^{2}-m_{r}\left(p-m_{r}\right)\right|$ obtained in the proof of Lemma 2.1 are sharp in their dependence on $p$ and $\mathbf{r}(\Sigma)$. Particularly this implies that without a further de-biasing step akin to the procedure in [11] it is impossible to improve the dependence on $p$ and $\mathbf{r}(\Sigma)$ in (2.4).

In principle Lemma 2.1 could be used to construct confidence sets and tests for $P_{r}$. However, in statistical applications $\mathbb{I}\left(P_{r}\right)$ is usually not known and one needs to estimate it from the data.

For this we use the plug-in estimator given by

$$
\hat{\mathbb{I}}\left(\hat{P}_{r}\right)^{1 / 2}:=\frac{1}{\sqrt{2}}\left[\hat{\Sigma}^{-1 / 2} \hat{C}_{r}^{-1} \otimes \hat{P}_{r} \hat{\Sigma}^{-1 / 2}+\hat{\Sigma}^{-1 / 2} \hat{P}_{r} \otimes \hat{C}_{r}^{-1} \hat{\Sigma}^{-1 / 2}\right]
$$

where we denote $\hat{C}_{r}^{-1}=\hat{\lambda}_{r} I-\hat{\Sigma}$. To be able to consistenly estimate $\lambda_{\text {min }}$ we need to assume that it is of larger order than $\|\hat{\Sigma}-\Sigma\| \asymp \sqrt{\mathbf{r}(\Sigma) / n}$. Our main result is then following Berry-Essen type Theorem:

Theorem 2.1. Suppose that $\boldsymbol{r}(\Sigma)=o(n)$, that $\mathbb{E}\|\hat{\Sigma}-\Sigma\| \leq(1-\gamma) \bar{g}_{r} / 2$ for some $\gamma \in(0,1)$, that $\bar{g}_{r}>\bar{c}$ for some constant $\bar{c}>0$ large enough and that for another large enough constant $c>0$

$$
\lambda_{\min } \geq c \sqrt{\frac{\mathbf{r}(\Sigma) \bigvee \log (p)}{n}} .
$$

Then, for $\Phi$ denoting the distribution function of a standard Gaussian random variable we have that

$$
\begin{aligned}
& \sup _{x \in \mathbb{R}}\left|\mathbb{P}\left(\frac{n\left\|\hat{\mathbb{I}}\left(\hat{P}_{r}\right)^{1 / 2}\left(\hat{P}_{r}-P_{r}\right)\right\|_{F}^{2}-m_{r}\left(p-m_{r}\right)}{\sqrt{2 m_{r}\left(p-m_{r}\right)}} \leq x\right)-\Phi(x)\right| \\
& \leq C\left(\gamma, \bar{g}_{r}, m_{r},\|\Sigma\|, \lambda_{r}\right)\left[\frac{1}{\sqrt{p}}+\frac{r(\Sigma)^{2} \vee \log (p)^{2}}{n \sqrt{p} \lambda_{\min }^{3}}+\sqrt{\frac{p \log (p)}{n}}\right. \\
& \left.\quad+\frac{(\mathbf{r}(\Sigma) \vee \log (p)) \sqrt{\log (p)}}{\lambda_{\min } \sqrt{n p}}+\frac{\sqrt{p} \boldsymbol{r}(\Sigma)}{n}\right] .
\end{aligned}
$$

All quantities except $P_{r}$ in the Wald statistic above are known or, as in the case of $m_{r}$, can assumed to be known (see Lemma 2.2 in [12]). This allows the construction of statistical tests and confidence ellipsoids for $P_{r}$. 
Considering the spiked covariance model (1.1) we have that $\mathbf{r}(\Sigma) \asymp p$ and $\lambda_{\min } \asymp 1$ and thus one can meaningfully apply Theorem 2.1 if $\mathbf{r}(\Sigma)=o\left(n^{2 / 3}\right)$. If $\lambda_{\min }$ shrinks to 0 the sample size requirements are becoming worse. For example, for models with decaying eigenvalues such that $\lambda_{i} \asymp i^{-\alpha}, 0 \leq \alpha<1$ we have that $\mathbf{r}(\Sigma) \asymp p^{1-\alpha}$ and $\lambda_{\min } \asymp p^{-\alpha}$. Therefore the application of Theorem 2.1 is feasible if $p^{3 / 2+\alpha}=o(n)$.

In case of the spiked covariance model the conditions of Theorem 2.1 compare favorably to the bootstrap approach used by [14] who need to assume that $\mathbf{r}(\Sigma)=o\left(n^{1 / 3}\right)$. Moreover, for models with decaying eigenvalues with $\alpha<3 / 8$ their condition is worse than our requirement $p^{3 / 2+\alpha}=o(n)$ whereas for $\alpha>3 / 8$ their condition is better.

The construction proposed in [10] and [11] requires no assumption on $\lambda_{\min }$ and allows for $\mathbf{r}(\Sigma)=o(n)$, but instead relies on sample splitting into three samples, assumes that $m_{r}=1$ and that $\|\Sigma\|_{F}^{2} \rightarrow \infty$. The last condition makes their Theorem unfeasible for application to models with quickly decaying eigenvalues $\lambda_{i} \asymp i^{-\alpha}, \alpha>1 / 2$.

\section{Proofs}

We first collect a few results from [8] and [9] which we will frequently use throughout our proof. The first Lemma is a perturbation bound for spectral projectors proven in [8].

Lemma 3.1. Suppose that $\tilde{\Sigma}=\Sigma+E$. Let $\tilde{P}_{r}$ be the spectral projector corresponding to the eigenvalues $\left\{\mu_{j}(\tilde{\Sigma}), j \in \Delta_{r}\right\}$. Then the following bound holds

$$
\left\|\tilde{P}_{r}-P_{r}\right\| \leq 4 \frac{\|E\|}{\bar{g}_{r}} .
$$

Moreover,

$$
\tilde{P}_{r}-P_{r}=L_{r}(E)+S_{r}(E)
$$

where $L_{r}(E)=C_{r} E P_{r}+P_{r} E C_{r}$ and where $C_{r}$ denotes the resolvent operator

$$
C_{r}=\sum_{s \neq r} \frac{P_{s}}{\lambda_{r}-\lambda_{s}}
$$

and where the remainder term can be bounded

$$
\left\|S_{r}(E)\right\| \leq 14\left(\frac{\|E\|}{\bar{g}_{r}}\right)^{2} .
$$

In the course of our proofs we will also need a finer analysis of the non-linear term $S_{r}(E)$. 
Lemma 3.2. The following bound holds

$$
S_{r}(E)=Z_{r}(E)+R_{r}(E)
$$

where

$Z_{r}(E)=P_{r} E C_{r} E C_{r}+C_{r} E C_{r} E P_{r}+C_{r} E P_{r} E C_{r}-P_{r} E P_{r} E C_{r}^{2}-P_{r} E C_{r}^{2} E P_{r}-C_{r}^{2} E P_{r} E P_{r}$

and where the third order remainder term is symmetric and fulfills

$$
\left\|R_{r}(E)\right\| \leq 72\left(\frac{\|E\|}{\bar{g}_{r}}\right)^{3} .
$$

Proof. The first part and symmetry of $R_{r}(E)$ follows immediately by inspecting the proof of Lemma 5 in [11]. Moreover,

$$
R_{r}(E)=-\frac{1}{2 \pi i} \oint_{\gamma_{r}} \sum_{k \geq 3}(-1)^{k}\left(R_{\Sigma}(\eta) E\right)^{k} R_{\Sigma}(\eta) d \eta
$$

where $\gamma_{r}$ denotes the circle of radius $\bar{g}_{r} / 2$ centred at $\lambda_{r}$ with counterclockwise orientation and $R_{\Sigma}(\eta)$ denotes the resolvent of $P_{r}$, i.e.

$$
R_{\Sigma}(\eta)=\sum_{j \geq 1} \frac{P_{j}}{\mu_{j}-\eta}
$$

Assume first that $\|E\| \leq \bar{g}_{r} / 3$. Then we have that

$$
\begin{aligned}
\left\|-\frac{1}{2 \pi i} \oint_{\gamma_{r}} \sum_{k \geq 3}(-1)^{k}\left(R_{\Sigma}(\eta) E\right)^{k} R_{\Sigma}(\eta) d \eta\right\| & \leq 2 \pi \frac{\bar{g}_{r}}{2}\left(\frac{2}{\bar{g}_{r}}\right)^{4}\|E\|^{3} \sum_{k=0}^{\infty}\left(\frac{2\|E\|}{\bar{g}_{r}}\right)^{k} \\
& \leq 24\left(\frac{\|E\|}{\bar{g}_{r}}\right)^{3} .
\end{aligned}
$$

If $\|E\| \geq \bar{g}_{r} / 3$ then by Lemma 3.1 and the explicit representation of the second order pertubation term in Lemma 5 in [11] we obtain that

$$
\begin{aligned}
& \left\|-\frac{1}{2 \pi i} \oint_{\gamma_{r}} \sum_{k \geq 3}(-1)^{k}\left(R_{\Sigma}(\eta) E\right)^{k} R_{\Sigma}(\eta) d \eta\right\| \\
\leq & \left\|\hat{P}_{r}-P_{r}\right\|+\left\|L_{r}(E)\right\|+\left\|-\frac{1}{2 \pi i} \oint_{\gamma_{r}}\left(R_{\Sigma}(\eta) E\right)^{2} R_{\Sigma}(\eta) d \eta\right\| \\
\leq & 4 \frac{\|E\|}{\bar{g}_{r}}+\frac{2\|E\|}{\bar{g}_{r}}+\frac{6\|E\|^{2}}{\bar{g}_{r}} \leq 72\left(\frac{\|E\|}{\bar{g}_{r}}\right)^{3}
\end{aligned}
$$

To bound $\|\hat{\Sigma}-\Sigma\|$ we will frequently use the following bound and concentration inequality obtained by Koltchinskii and Lounici in [9]. 
Theorem 3.1. Let $X_{1}, \ldots, X_{n}$ be i.i.d. centred Gaussian random vectors with covariance matrix $\Sigma$ and such that $\mathbb{E}\left\|X_{1}\right\|^{2}<\infty$. Suppose that $r(\Sigma)=o(n)$. Then, for some constant $C_{q}>0$

$$
\left(\mathbb{E}\|\hat{\Sigma}-\Sigma\|^{q}\right)^{1 / q} \leq C_{q}\|\Sigma\| \sqrt{\frac{\boldsymbol{r}(\Sigma)}{n}}
$$

Moreover, there exists another constant $C^{\prime}>0$ such that for all $t \geq 1$ with probability at least $1-e^{-t}$ we have that,

$$
|\|\hat{\Sigma}-\Sigma\|-\mathbb{E}\|\hat{\Sigma}-\Sigma\|| \leq C^{\prime}\|\Sigma\|\left(\sqrt{\frac{t}{n}} \bigvee \frac{t}{n}\right)
$$

In the following we denote $E=\hat{\Sigma}-\Sigma, L_{r}:=L_{r}(E), S_{r}:=S_{r}(E), Z_{r}:=$ $Z_{r}(E)$ and $R_{r}:=R_{r}(E)$. We now turn to the proof of Lemma 2.1.

Proof of Lemma 2.1. Going line by line through the proofs of Lemma 5, Theorem 5 and the calculations leading to display (5.17) in [10] it is easy to see that one can adjust them to show

$$
\frac{n\left[\left\|\mathbb{I}\left(P_{r}\right)^{1 / 2}\left(\hat{P}_{r}-P_{r}\right)\right\|_{F}^{2}-\mathbb{E}\left\|\mathbb{I}\left(P_{r}\right)^{1 / 2}\left(\hat{P}_{r}-P_{r}\right)\right\|_{F}^{2}\right]}{\sqrt{2 m_{r}\left(p-m_{r}\right)}} \stackrel{d}{=} \frac{\sum_{i=1}^{m_{r}\left(p-m_{r}\right)}\left(g_{i}^{2}-1\right)}{\sqrt{2 m_{r}\left(p-m_{r}\right)}}+Z_{n}^{\prime},
$$

where $Z_{n}^{\prime}$ fulfills with probability at least $1-e^{-t}$ for every $1 \leq t \leq n$

$$
\left|Z_{n}^{\prime}\right| \leq C^{\prime}\left(\gamma, \bar{g}_{r}, m_{r},\|\Sigma\|, \lambda_{r}\right)\left(\frac{t}{\sqrt{n}} \bigvee \frac{(\mathbf{r}(\Sigma) \vee t) \sqrt{t}}{\lambda_{\min } \sqrt{n p}} \bigvee \sqrt{\frac{p}{n}} \sqrt{t} \bigvee \frac{t^{3 / 2}}{\sqrt{n p}}\right)
$$

Thus it remains to obtain a tight bound for $\mathbb{E}\left\|\mathbb{I}\left(P_{r}\right)^{1 / 2}\left(\hat{P}_{r}-P_{r}\right)\right\|_{F}^{2}$. Using decomposition (3.2) we obtain that

$\mathbb{E}\left\|\mathbb{I}\left(P_{r}\right)^{1 / 2}\left(\hat{P}_{r}-P_{r}\right)\right\|_{F}^{2}=\left\|\mathbb{I}\left(P_{r}\right)^{1 / 2} L_{r}\right\|_{F}^{2}+\left\|\mathbb{I}\left(P_{r}\right)^{1 / 2} S_{r}\right\|_{F}^{2}+\operatorname{tr}\left(\left(\mathbb{I}\left(P_{r}\right)^{1 / 2} L_{r}\right)\left(\mathbb{I}\left(P_{r}\right)^{1 / 2} S_{r}\right)\right)$.

As in the proof of Theorem 5 in [10] we obtain that

$$
n \mathbb{E}\left\|\mathbb{I}\left(P_{r}\right)^{1 / 2} L_{r}\right\|_{F}^{2}=n \mathbb{E}\left\|P_{r}^{\perp}(\Sigma)^{-1 / 2} E(\Sigma)^{-1 / 2} P_{r}\right\|_{F}^{2}=m_{r}\left(p-m_{r}\right) .
$$

Moreover, the second term in (3.12) can be bound by applying Lemma 3.1 and Theorem 3.1 as follows

$$
\mathbb{E}\left\|\mathbb{I}\left(P_{r}\right)^{1 / 2} S_{r}\right\|_{F}^{2} \leq 2 m_{r} \mathbb{E}\left\|C_{r}^{-1} \Sigma^{-1 / 2} S_{r} P_{r} \Sigma^{-1 / 2}\right\| \lesssim \frac{2 m_{r}\|\Sigma\|^{2}}{\bar{g}_{r}^{4} \lambda_{r} \lambda_{\min }} \mathbb{E}\|E\|^{4}=O\left(\frac{\mathbf{r}(\Sigma)^{2}}{n^{2} \lambda_{\min }}\right) .
$$

For the last remainder term in (3.12) the naive use of Cauchy-Schwarz does not suffice and and we have to use higher-order pertubation theory to obtain good 
enough bounds. Applying Lemma 3.2 and using symmetry of $L_{r}, R_{r}, Z_{r}$ and $\mathbb{I}\left(P_{r}\right)^{1 / 2}$ we obtain that,

$$
\begin{aligned}
& \mathbb{E}\left\langle\mathbb{I}\left(P_{r}\right)^{1 / 2} L_{r}, \mathbb{I}\left(P_{r}\right)^{1 / 2} S_{r}\right\rangle=\mathbb{E}\left\langle\Sigma^{-1} P_{r} E P_{r}^{\perp} \Sigma^{-1}, P_{r} E C_{r} E P_{r}^{\perp}-P_{r} E P_{r} E C_{r}\right\rangle \\
& +\mathbb{E}\left\langle\Sigma^{-1} P_{r} E P_{r}^{\perp} \Sigma^{-1}, P_{r} R_{r}(E) C_{r}^{-1}\right\rangle .
\end{aligned}
$$

We now bound the three terms in (3.13) separately. For $\left\{\theta_{j}\right\}_{j \in \Delta_{r}}$ denoting the eigenvectors of an eigen-decomposition of $P_{r}\left\langle X_{1}, \theta_{j}\right\rangle$ and $P_{r}^{\perp} X_{1}$ are independent. Thus, we obtain that the first term in (3.13) equals

$$
\begin{aligned}
& \mathbb{E}\left\langle\Sigma^{-1} P_{r} E P_{r}^{\perp} \Sigma^{-1}, P_{r} E C_{r} E P_{r}^{\perp}\right\rangle \\
= & \left.\frac{1}{n^{2}} \sum_{j \in \Delta_{r}} \operatorname{tr}\left(\Sigma^{-1} P_{r}^{\perp} X_{1} X_{1}^{T} \theta_{j} \theta_{j}^{T} \Sigma^{-1} \theta_{j} \theta_{j}^{T} X_{1} X_{1}^{T} C_{r}\left(X_{1} X_{1}^{T}-\Sigma\right) P_{r}^{\perp}\right)\right) \\
= & \frac{m_{r}}{n^{2}} \sum_{i \neq l, i, l \notin \Delta_{r}} \frac{1}{\lambda_{i}\left(\lambda_{r}-\lambda_{l}\right)} \mathbb{E} \operatorname{tr}\left[\theta_{i} \theta_{i}^{T} X_{1} X_{1}^{T} \theta_{l} \theta_{l}^{T} X_{1} X_{1}^{T} \theta_{i} \theta_{i}^{T}\right] \\
& +\frac{m_{r}}{n^{2}} \sum_{i \notin \Delta_{r}} \frac{1}{\lambda_{i}\left(\lambda_{r}-\lambda_{i}\right)} \mathbb{E} \operatorname{tr}\left[\theta_{i} \theta_{i}^{T} X_{1} X_{1}^{T} \theta_{i} \theta_{i}^{T}\left(X_{1} X_{1}^{T}-\Sigma\right) \theta_{i} \theta_{i}^{T}\right] \\
= & \frac{m_{r}}{n^{2}}\left[\sum_{i \neq l, i, l \notin \Delta_{r}} \frac{1}{\lambda_{i}\left(\lambda_{r}-\lambda_{l}\right)} \mathbb{E}\left\langle X_{1}, \theta_{i}\right\rangle^{2} \mathbb{E}\left\langle\theta_{l}, X_{1}\right\rangle^{2}+\sum_{i \notin \Delta_{r}} \frac{1}{\lambda_{i}\left(\lambda_{r}-\lambda_{i}\right)}\left(\mathbb{E}\left\langle X_{1}, \theta_{i}\right\rangle^{4}-\mathbb{E}\left\langle X_{1}, \theta_{i}\right\rangle^{2} \lambda_{i}\right)\right] \\
= & \frac{m_{r}}{n^{2}}\left[\sum_{i \neq l, i, l \notin \Delta_{r}} \frac{\lambda_{l}}{\lambda_{r}-\lambda_{l}}+\sum_{i \neq \Delta_{r}} \frac{2 \lambda_{i}}{\lambda_{r}-\lambda_{i}}\right] \lesssim \frac{m_{r}}{\bar{g}_{r}} \frac{p \mathbf{r}(\Sigma)}{n^{2}}
\end{aligned}
$$

The second term in (3.13) can be estimated similarly,

$$
\begin{aligned}
& \frac{1}{n^{2} \lambda_{r}} \sum_{j \in \Delta_{r}} \mathbb{E} \operatorname{tr}\left(\Sigma^{-1} P_{r}^{\perp} X_{1} X_{1}^{T} \theta_{j} \theta_{j}^{T}\left(X_{1} X_{1}^{T}-\Sigma\right) \theta_{j} \theta_{j}^{T} X_{1} X_{1}^{T} C_{r}\right) \\
= & \frac{2 m_{r} \lambda_{r}}{n^{2}} \mathbb{E} \operatorname{tr}\left(P_{r}^{\perp} \Sigma^{-1} X_{1} X_{1}^{T} C_{r}\right) \\
= & \frac{2 m_{r} \lambda_{r}}{n^{2}} \sum_{i \notin \Delta_{r}} \frac{\mathbb{E}\left\langle\theta_{i}, X_{1}\right\rangle^{2}}{\lambda_{i}\left(\lambda_{r}-\lambda_{i}\right)} \lesssim \frac{m_{r}\|\Sigma\|}{\bar{g}_{r}} \frac{p}{n^{2}} .
\end{aligned}
$$

The last term can be bound using Cauchy Schwarz, Lemma 3.2 and 3.1,

$$
\begin{aligned}
& \mathbb{E}\left\langle\Sigma^{-1} P_{r} E P_{r}^{\perp} \Sigma^{-1 / 2}, P_{r} R_{r}(E) C_{r}^{-1}\right\rangle \\
\leq & \frac{\|\Sigma\| \sqrt{m_{r}}}{\sqrt{\lambda_{r} \lambda_{\min }}} \sqrt{\mathbb{E}\left\|P_{r} \Sigma^{-1 / 2} E \Sigma^{-1 / 2} P_{r}^{\perp}\right\|_{F}^{2}} \sqrt{\mathbb{E}\left\|R_{r}(E)\right\|^{2}} \\
\lesssim & \frac{\|\Sigma\| m_{r}}{\bar{g}_{r}^{3} \sqrt{\lambda_{r} \lambda_{\min }}} \sqrt{\frac{p}{n}}\left(\mathbb{E}\|E\|^{6}\right)^{1 / 2} \lesssim \frac{\|\Sigma\|^{4} m_{r}}{\bar{g}_{r}^{3} \sqrt{\lambda_{r}}} \frac{\sqrt{p} \mathbf{r}(\Sigma)^{3 / 2}}{n^{2} \sqrt{\lambda_{\min }}}
\end{aligned}
$$


Thus, summarizing, we have that

$$
\frac{n \mathbb{E}\left\|\mathbb{I}\left(P_{r}\right)^{-1 / 2}\left(\hat{P}_{r}-P_{r}\right)\right\|_{F}^{2}}{\sqrt{2 m_{r}\left(p-m_{r}\right)}}=\frac{m_{r}\left(p-m_{r}\right)}{\sqrt{2 m_{r}\left(p-m_{r}\right)}}+O\left(\frac{\sqrt{p} \mathbf{r}(\Sigma)}{n}+\frac{\mathbf{r}(\Sigma)^{2}}{n \sqrt{p} \lambda_{\min }}\right),
$$

and the claim follows.

We now turn to the proof of Theorem 2.1 and show first that we can replace $\mathbb{I}\left(P_{r}\right)^{1 / 2}$ by $\hat{\mathbb{I}}\left(\hat{P}_{r}\right)^{1 / 2}$.

Proof of Theorem 2.1. We have that

$$
\begin{aligned}
& \left\|\left(\mathbb{I}\left(P_{r}\right)^{1 / 2}-\hat{\mathbb{I}}\left(\hat{P}_{r}\right)^{1 / 2}\right)\left(\hat{P}_{r}-P_{r}\right)\right\|_{F} \\
\leq & 2 \sqrt{m_{r}}\left\|\hat{P}_{r}-P_{r}\right\|\left\|\hat{C}_{r}^{-1}\right\|\left\|\hat{\Sigma}^{-1 / 2}\right\|\left(\left|\frac{1}{\sqrt{\hat{\lambda}_{r}}}-\frac{1}{\sqrt{\lambda_{r}}}\right|+\frac{\left\|\hat{P}_{r}-P_{r}\right\|}{\sqrt{\lambda_{r}}}\right) \\
& +\frac{2 \sqrt{m_{r}}}{\sqrt{\lambda_{r}}}\left\|\hat{P}_{r}-P_{r}\right\|\left(\left\|\hat{C}_{r}^{-1}\right\|\left\|\hat{\Sigma}^{-1 / 2}-\Sigma^{-1 / 2}\right\|+\left\|C_{r}^{-1}-\hat{C}_{r}^{-1}\right\|\left\|\Sigma^{-1 / 2}\right\|\right) \\
= & I+I I+I I I+I V .
\end{aligned}
$$

We now bound each of these four terms separately. We have that

$$
\begin{aligned}
I & \leq 8 \sqrt{m_{r}} \frac{\|E\|}{\bar{g}_{r}}\|\Sigma+E\|\left|\hat{\lambda}_{\min }\right|^{-1 / 2} \sum_{k=1}^{\infty} \frac{\left|\hat{\lambda}_{r}-\lambda_{r}\right|^{k}}{\lambda_{r}^{k+1 / 2}} \\
& \leq 16 \sqrt{m_{r}} \frac{\|E\|}{\bar{g}_{r}}\|\Sigma\|\left|\lambda_{\min }-\|E\|\right|^{-1 / 2} \sum_{k=1}^{\infty} \frac{\|E\|^{k}}{\lambda_{r}^{k+1 / 2}} \lesssim \frac{\sqrt{m_{r}}\|\Sigma\|^{3}}{\bar{g}_{r} \sqrt{\lambda_{r}}} \frac{\mathbf{r}(\Sigma) \vee t}{n \sqrt{\lambda_{\min }}}
\end{aligned}
$$

with probability at least $1-e^{-t}$ for $1 \leq t \leq \log (p)$ and where we used Theorem 3.1 to bound $\|E\|$, Lidski's inequality to bound $\left|\hat{\lambda}_{r}-\lambda_{r}\right|$ and the $\lambda_{\min }$ condition (2.6) to bound $\lambda_{\min }-\|E\| \geq \lambda_{\min } / 2$. The second term can be bounded likewise, i.e. on the same event as the bound for $I$ we have with probability at least $1-e^{-t}$ for $1 \leq t \leq \log (p)$ that

$$
I I \leq \frac{32 \sqrt{m_{r}+m_{r}}}{\sqrt{\lambda_{r}}} \frac{\|E\|^{2}}{\bar{g}_{r}^{2}}\|\hat{\Sigma}\|\left\|\hat{\Sigma}^{-1 / 2}\right\| \lesssim \frac{\sqrt{m_{r}}\|\Sigma\|^{3}}{\bar{g}_{r}^{2} \sqrt{\lambda_{r}}} \frac{\mathbf{r}(\Sigma) \vee t}{n \sqrt{\lambda_{\min }}} .
$$

Using matrix series expansions of $\Sigma \hat{\Sigma}^{-1}$ and of $\left(\Sigma \hat{\Sigma}^{-1}\right)^{1 / 2}$ around $I$ we can bound the third term on the same event. We have with probability at least $1-e^{-t}$ for $1 \leq t \leq \log (p)$ :

$$
\begin{aligned}
& I I I \leq \frac{8 \sqrt{m_{r}}\|\hat{\Sigma}\|\|E\|}{\bar{g}_{r} \sqrt{\lambda_{r}}}\left\|\hat{\Sigma}^{-1 / 2}-\Sigma^{-1 / 2}\right\| \\
& \lesssim \frac{\sqrt{m_{r}}\|\hat{\Sigma}\|\|E\|}{\bar{g}_{r} \sqrt{\lambda_{\min } \lambda_{r}}} \sum_{j=1}^{\infty}\left(\sum_{k=1}^{\infty}\left\|\Sigma^{-1}\right\|^{k}\|E\|^{k}\right)^{j} \\
& \lesssim \frac{\sqrt{m_{r}}\|\Sigma\|^{3}}{\bar{g}_{r} \sqrt{\lambda_{r}}} \frac{\mathbf{r}(\Sigma) \vee t}{n \lambda_{\text {min }}^{3 / 2}}
\end{aligned}
$$


where we used again the $\lambda_{\min }$ condition (2.6) to ensure convergence of the series. Finally, the fourth term can be bound in the same fashion on the same event. For $1 \leq t \leq \log (p)$ with probability at least $1-e^{-t}$ we have that

$$
I V \leq \frac{8 \sqrt{2 m_{r}}\|E\|}{\bar{g}_{r} \sqrt{\lambda_{r} \lambda_{\min }}}\left(\left|\hat{\lambda}_{r}-\lambda_{r}\right|+\|E\|\right) \lesssim \frac{m_{r}\|\Sigma\|^{2}}{\bar{g}_{r} \sqrt{\lambda_{r}}} \frac{\mathbf{r}(\Sigma) \vee t}{n \sqrt{\lambda_{\min }}}
$$

Thus, summarizing, and since we bounded $I, I I, I I I$ and $I V$ on the same event we have, choosing $t=\log (p)$ with probability at least $1-1 / p$ that

$$
\frac{n\left\|\left(\mathbb{I}\left(P_{r}\right)^{1 / 2}-\hat{\mathbb{I}}\left(P_{r}\right)^{1 / 2}\right)\left(\hat{P}_{r}-P_{r}\right)\right\|_{F}^{2}}{\sqrt{2 m_{r}\left(p-m_{r}\right)}} \leq C\left(\|\Sigma\|, \lambda_{r}, m_{r}, \bar{g}_{r}\right) \frac{\mathbf{r}(\Sigma)^{2} \vee \log (p)^{2}}{n \sqrt{p} \lambda_{\min }^{3}} .
$$

Defining for random variables $\eta$ and $\xi \Delta(\eta, \xi):=\sup _{x \in \mathbb{R}}|\mathbb{P}(\xi \leq x)-\mathbb{P}(\eta \leq x)|$ the anti-concentration bound in Lemma 4.6. from [12] combined with (3.22) and Lemma 2.1 thus implies that for $\eta:=\frac{n\left\|\hat{\mathbb{1}}\left(P_{r}\right)^{1 / 2}\left(\hat{P}_{r}-P_{r}\right)\right\|_{F}^{2}-m_{r}\left(p-m_{r}\right)}{\sqrt{2 m_{r}\left(p-m_{r}\right)}}$ and $Z$ denoting a standard Gaussian random variable we have that,

$$
\begin{aligned}
\Delta(\eta, Z) \leq & \Delta(\xi, Z)+\frac{1}{p} \bigvee C\left(\gamma, \bar{g}_{r}, m_{r},\|\Sigma\|, \lambda_{r}\right)\left[\frac{\mathbf{r}(\Sigma)^{2} \vee \log (p)^{2}}{n \sqrt{p} \lambda_{\min }^{3}}+\sqrt{\frac{p \log (p)}{n}}\right. \\
& \left.+\frac{(\mathbf{r}(\Sigma) \vee \log (p)) \sqrt{\log (p)}}{\lambda_{\min } \sqrt{n p}}+\frac{\sqrt{p} \mathbf{r}(\Sigma)}{n}\right]
\end{aligned}
$$

where the main term $\xi$ is defined as $\sum_{i=1}^{m_{r}\left(p-m_{r}\right)}\left(g_{i}^{2}-1\right) / \sqrt{2 m_{r}\left(p-m_{r}\right)}$. Theorem 2.1 now follows from the bound above and the Berry-Essen Theorem applied to $\Delta(\xi, Z)$.

\section{References}

[1] T. W. Anderson. (1963). Asymptotic theory for principal component analysis. Ann. Math. Statist., 34 122-148 MR0145620

[2] Q. Berthet and P. Rigollet. (2013). Optimal detection of sparse principal components in high dimension. Ann. Statist., 41(4), 1780-1815. MR3127849

[3] T. Cai, Z. Ma, and Y. Wu. (2013). Sparse PCA: optimal rates and adaptive estimation. Ann. Statist., 41(6): 3074-3110 MR3161458

[4] M.L. Eaton. (1983). Multivariate Statistics: A Vector Space Approach. John Wiley \& Sons, Inc., New York MR0716321

[5] C. Gao and H. Zhou. (2015). Rate-optimal posterior contraction for sparse PCA. Ann. Statist. 43(2), 785-818 MR3325710

[6] I. M. Johnstone. (2001). On the distribution of the largest eigenvalue in principal components analysis. Ann. Statist., 29(2), 295-327 MR1863961

[7] I. M. Johnstone and A.Y. Lu. (2009). On consistency and sparsity for principal components analysis in high dimensions. J. Amer. Statist. Assoc. 104 682-693. MR2751448 
[8] V. Koltchinskii and K. Lounici. (2016). Asymptotics and concentration bounds for bilinear forms of spectral projectors of sample covariance. Ann. Inst. Henri Poincar Probab. Stat., 52(4), 1976-2013. MR3573302

[9] V. Koltchinskii and K. Lounici. (2017). Concentration inequalities and moment bounds for sample covariance operators. Bernoulli, 23(1), 110-133. MR3556768

[10] ) V. Koltchinskii and K. Lounici (2017). Normal approximation and concentration of spectral projectors of sample covariance Ann. Statist., 45(1), 121-157. MR3611488

[11] V. Koltchinskii and K. Lounici (2017). New asymptotic results in principal component analysis. Sankhya A 79(2): 254-297

[12] V. Koltchinskii, M. Löffler and R. Nickl. (2017). Efficient Estimation of Linear Functionals of Principal Components Preprint at https://arxiv.org/abs/1708.076429

[13] B. Nadler. (2008). Finite sample approximation results for principal component analysis: a matrix perturbation approach. Ann. Statist., 36(6), 27912817. MR2485013

[14] A. Naumov, V. Spokoiny and V. Ulyanov. (2017). Bootstrap confidence sets for spectral projectors of sample covariance. Preprint at https://arxiv.org/pdf/1703.00871.pdf

[15] D. Paul. (2007). Asymptotics of Sample Eigenstructure for a Large Dimensional Spiked Covariance Model. Statistica Sinica., 17(4), 1617-1642. MR2399865

[16] M. Reiss and M. Wahl. (2016). Non-asymptotic upper bounds for the reconstruction error of PCA. Preprint at https://arxiv.org/abs/1609.03779

[17] I. Silin and V. Spokoiny. (2017). Bayesian inference for spectral projectors of covariance matrix Preprint at https://arxiv.org/abs/1711.11532

[18] V. Vu and J. Lei. (2013). Minimax sparse principal subspace estimation in high dimensions. Ann. Statist., 41(6), 2905-2947. MR161452

[19] A. Wald. (1943). Tests of Statistical Hypotheses Concerning Several Parameters When the Number of Observations is Large. Transactions of the American Mathematical Society., 54(3), 426-482.

[20] T. Wang, Q. Berthet and R.J Samworth. (2016). Statistical and Computational Trade-offs in estimation of sparse principal components. Ann. Statist., 44(5), 1896-1930. MR3546438

[21] W. Wang and J. Fan. (2017). Asymptotics of empirical eigenstructure for high dimensional spiked covariance. Ann. Statist., 45(3), 1342-1374. MR3662457 